\title{
Analytic smoothing effect for system of nonlinear Schrödinger equations with general mass resonance
}

\author{
Takayoshi Ogawa and Takuya SAto \\ (Received January 19, 2019) \\ (Revised October 21, 2019)
}

\begin{abstract}
We prove the analytic smoothing effect for solutions to the system of nonlinear Schrödinger equations under the gauge invariant nonlinearities. This result extends the known result due to Hoshino [Nonlinear Differential Equations Appl. 24 (2017), Art. 62]. Under rapidly decaying condition on the initial data, the solution shows a smoothing effect and is real analytic with respect to the space variable. Our theorem covers not only the case for the gauge invariant setting but also multiple component case with higher power nonlinearity up to the fifth order.
\end{abstract}

\section{Introduction}

We consider the Cauchy problem of the following nonlinear Schrödinger equations:

$$
\begin{cases}i \partial_{t} u_{j}+\frac{1}{2 m_{j}} \Delta u_{j}=f_{j}(u, \bar{u}), & t \in \mathbb{R}, x \in \mathbb{R}^{n}, j=1,2, \ldots, k, k \in \mathbb{N}, \\ u_{j}(0)=\phi_{j}, & x \in \mathbb{R}^{n},\end{cases}
$$

where $m_{j}>0, f_{j}: \mathbb{C}^{k} \times \mathbb{C}^{k} \rightarrow \mathbb{C}, u=\left(u_{1}, \ldots, u_{k}\right): \mathbb{R} \times \mathbb{R}^{n} \rightarrow \mathbb{C}^{k}$ is the unknown function and $\phi=\left(\phi_{1}, \ldots, \phi_{k}\right): \mathbb{R}^{n} \rightarrow \mathbb{C}^{k}$ is a given initial data.

The nonlinear Schrödinger equation is classified as a dispersive type of partial differential equations and there is no smoothing effect that often appears in solutions to parabolic type equations. However if we restrict the initial data satisfying spacial weight condition, then one can find that solutions to the nonlinear Schrödinger equations exhibits a local smoothing effect (cf. Kato [14]). Hayashi-Saitoh [4] proved that if the initial data $\phi$ decays exponentially as $|x| \rightarrow \infty$, then there exists an analytic solution to the single nonlinear Schrödinger equation:

2010 Mathematics Subject Classification. 35Q55, 35B30.

Key words and phrases. Nonlinear Schrödinger equation, analytic smoothing effect, gauge invariance. 


$$
\begin{cases}i \partial_{t} u+\frac{1}{2 m} \Delta u=f(u), & t \in \mathbb{R}, x \in \mathbb{R}^{n}, \\ u(0)=\phi, & x \in \mathbb{R}^{n},\end{cases}
$$

where $f(u)=|u|^{2} u$. For the cases of general polynomial nonlinearities $f(u)$, it was shown by several authors that the solution has the analytic smoothing effect ([2], [4], [5], [8]-[13], [15], [16], [18]).

Hoshino-Ozawa [13] showed the analytic smoothing effect for the solution to the following nonlinear Schrödinger equations:

$$
\begin{cases}i \partial_{t} u_{1}+\frac{1}{2 m_{1}} \Delta u_{1}=f_{1}\left(u_{1}, u_{2}\right), & t \in \mathbb{R}, x \in \mathbb{R}^{n}, \\ i \partial_{t} u_{2}+\frac{1}{2 m_{2}} \Delta u_{2}=f_{2}\left(u_{1}, u_{2}\right), & t \in \mathbb{R}, x \in \mathbb{R}^{n}, \\ \left(u_{1}(0), u_{2}(0)\right)=\left(\phi_{1}, \phi_{2}\right), & x \in \mathbb{R}^{n},\end{cases}
$$

where the nonlinear coupling $\left(f_{1}, f_{2}\right)$ is given by either of the followings

$$
\begin{gathered}
\left\{\begin{array}{l}
f_{1}\left(u_{1}, u_{2}\right)=\overline{u_{1}} u_{2}, \quad \text { for } p=2, \\
f_{2}\left(u_{1}, u_{2}\right)=u_{1}^{2},
\end{array}\right. \\
\left\{\begin{array}{l}
f_{1}\left(u_{1}, u_{2}\right)=\bar{u}_{1}^{2} u_{2}+\left|u_{1}\right|^{2} u_{1}+\left|u_{2}\right|^{2} u_{1}, \quad \text { for } p=3, \\
f_{2}\left(u_{1}, u_{2}\right)=u_{1}^{3}+\left|u_{2}\right|^{2} u_{2}+\left|u_{1}\right|^{2} u_{2},
\end{array}\right. \\
\left\{\begin{array}{l}
f_{1}\left(u_{1}, u_{2}\right)=\bar{u}_{1}^{4} u_{2}+\left|u_{1}\right|^{4} u_{1}, \quad \text { for } p=5 . \\
f_{2}\left(u_{1}, u_{2}\right)=u_{1}^{5}+\left|u_{2}\right|^{4} u_{2},
\end{array}\right.
\end{gathered}
$$

The nonlinearities given by (1.3), (1.4), and (1.5) satisfy

$$
\left\{\begin{array}{l}
e^{i \theta} f_{1}\left(z_{1}, z_{2}\right)=f_{1}\left(e^{i \theta} z_{1}, e^{i p \theta} z_{2}\right), \\
e^{i p \theta} f_{2}\left(z_{1}, z_{2}\right)=f_{2}\left(e^{i \theta} z_{1}, e^{i p \theta} z_{2}\right)
\end{array}\right.
$$

for any $\theta \in \mathbb{R}$ and $z_{1}, z_{2} \in \mathbb{C}$.

We prove the analytic smoothing effect of the solution to (1.1) with the $p$-th powered nonlinearities $\left\{f_{j}(u, \bar{u})\right\}_{j=1,2, \ldots, k}$ satisfying a gauge invariance.

Definition 1. The nonlinear coupling functions $f_{j}: \mathbb{C}^{2 k} \rightarrow \mathbb{C}$ satisfy the gauge invariance if each $f_{j}(j=1, \ldots, k)$ satisfies for any $\theta \in \mathbb{R}$ and $w, z \in \mathbb{C}^{k}$,

$e^{i m_{j} \theta} f_{j}\left(w_{1}, \ldots, w_{k}, z_{1}, \ldots, z_{k}\right)=f_{j}\left(e^{i m_{1} \theta} w_{1}, \ldots, e^{i m_{k} \theta} w_{k}, e^{i m_{1} \theta} z_{1}, \ldots, e^{i m_{k} \theta} z_{k}\right)$

for some $m=\left(m_{1}, \ldots, m_{k}\right) \in \mathbb{Z}_{\geq 0}^{k}$. We call the condition (1.6) the mass resonance. 
We restrict the nonlinearity to the following form: for any $u=\left(u_{1}, \ldots, u_{k}\right)$, $v=\left(v_{1}, \ldots, v_{k}\right): \mathbb{R} \times \mathbb{R}^{n} \rightarrow \mathbb{C}^{k}$,

$$
f_{j}(u, v) \equiv \lambda_{j} \prod_{\ell=1}^{k} u_{\ell}^{\alpha_{j, \ell}} v_{\ell}^{\beta_{j, \ell}} \quad \text { for } j=1, \ldots, k,
$$

where $\lambda_{j} \in \mathbb{C}, \alpha_{j}=\left(\alpha_{j, 1}, \ldots, \alpha_{j, k}\right)$ and $\beta_{j}=\left(\beta_{j, 1}, \ldots, \beta_{j, k}\right) \in \mathbb{Z}_{\geq 0}^{k}$ satisfy

$$
\alpha_{j, 1}+\cdots+\alpha_{j, k}+\beta_{j, 1}+\cdots+\beta_{j, k}=p
$$

with $p \in \mathbb{N}$. In this paper, we consider the type of nonlinearity $f_{j}(u, \bar{u})$.

Proposition 1. Let $k, p \in \mathbb{N}$. The nonlinearity $f_{j}$ given by (1.7) and (1.8) satisfies the condition (1.6) if and only if given a set of constants $m=$ $\left(m_{1}, \ldots, m_{k}\right) \in \mathbb{Z}_{\geq 0}^{k}$, exponents $\alpha_{j}$ and $\beta_{j} \in \mathbb{Z}_{\geq 0}^{k}$ satisfy

$$
m_{j}=m_{1}\left(\alpha_{j, 1}-\beta_{j, 1}\right)+\cdots+m_{k}\left(\alpha_{j, k}-\beta_{j, k}\right)
$$

for any $j=1,2, \ldots, k$.

In the 2-component case, the following nonlinearity $\left(f_{1}, f_{2}\right)$ defined by

$$
f_{j}\left(u_{1}, u_{2}\right) \equiv\left|u_{1}\right|^{2} u_{1},\left|u_{1}\right|^{2} u_{2}, u_{1}^{2} \overline{u_{2}}, u_{1}\left|u_{2}\right|^{2}, \overline{u_{1}} u_{2}^{2},\left|u_{2}\right|^{2} u_{2} \quad \text { for } j=1,2
$$

satisfies the condition (1.6) with $m=(1,1), p=3$ and the nonlinearity

$$
\left\{\begin{array}{l}
f_{1}\left(u_{1}, u_{2}\right) \equiv \bar{u}_{1}^{2} u_{2}^{2} \\
f_{2}\left(u_{1}, u_{2}\right) \equiv u_{1}^{3} \overline{u_{2}}
\end{array}\right.
$$

satisfies (1.6) with $m=(2,3)$ and $p=4$.

In order to state our result, we introduce the following function spaces introduced by Hayashi-Ozawa [10] and Hoshino-Ozawa [12]. For any $m>0$ and $\alpha \in \mathbb{N}$,

$$
\begin{aligned}
& L_{\exp , m}^{2}\left(\mathbb{R}^{n}\right) \equiv\left\{f \in L^{2}\left(\mathbb{R}^{n}\right) ;\|f\|_{L_{\text {exp }, m}^{2}\left(\mathbb{R}^{n}\right)} \equiv \sup _{a \in B_{1}(0)}\left\|e^{m a \cdot x} f\right\|_{L^{2}}<\infty\right\}, \\
& H_{\exp , m}^{\alpha}\left(\mathbb{R}^{n}\right) \equiv\left\{f \in H^{\alpha}\left(\mathbb{R}^{n}\right) ;\|f\|_{H_{\text {exp }, m}^{\alpha}\left(\mathbb{R}^{n}\right)} \equiv \sup _{a \in B_{1}(0)}\left\|e^{m a \cdot x} f\right\|_{H^{\alpha}}<\infty\right\},
\end{aligned}
$$

where $L^{2}=L^{2}\left(\mathbb{R}^{n}\right)$ and $H^{\alpha}=H^{\alpha}\left(\mathbb{R}^{n}\right)$ are the Lebesgue and the Sobolev spaces, respectively and $B_{1}(0)=\left\{x \in \mathbb{R}^{n} ;|x|<1\right\}$. Let $L^{\theta}\left(I ; L^{q}\left(\mathbb{R}^{n}\right)\right)$ be the Bochner space where $I \subset \mathbb{R}$ is an open interval. We call $L^{\theta}\left(I ; L^{q}\left(\mathbb{R}^{n}\right)\right)$ the Strichartz space if a pair of exponents $(\theta, q)$ is admissible as

$$
\frac{n}{2}=\frac{n}{q}+\frac{2}{\theta}
$$


with

$$
\begin{cases}2 \leq q \leq \frac{2 n}{n-2}, & n \geq 3 \\ 2 \leq q<\infty, & n=2 \\ 2 \leq q \leq \infty, & n=1\end{cases}
$$

Let

$$
J_{m}(t) \equiv x+\frac{i t}{m} \nabla
$$

be the generator of the Galilei transform. The following operator $e^{m a \cdot J_{m}}$ has been introduced in [10], [12] and Hoshino [7]: For $a \in B_{1}(0)$,

$$
e^{m a \cdot J_{m}(t)} f \equiv \sum_{k=0}^{\infty} \frac{m^{k}}{k !}\left(a \cdot J_{m}(t)\right)^{k} f
$$

with the domain

$$
D\left(e^{m a \cdot J_{m}(t)}\right)=\left\{f \in \mathscr{S}\left(\mathbb{R}^{n}\right) ; \sup _{a \in B_{1}(0)}\left\|e^{m a \cdot J_{m}(t)} f\right\|_{L^{q}}<\infty\right\}
$$

for any $t \neq 0$ and $q \geq 2$. For any $t \neq 0, e^{m a \cdot J_{m}}$ is represented as

$$
e^{m a \cdot J_{m}} f=e^{i(t / 2 m) \Delta} e^{m a \cdot x} e^{-i(t / 2 m) \Delta} f,
$$

and

$$
e^{m a \cdot J_{m}(t)} f=e^{i\left(m|x|^{2} / 2 t\right)} e^{i t a \cdot \nabla} e^{i\left(-m|x|^{2} / 2 t\right)} f
$$

where

$$
e^{i t a \cdot \nabla} f=\mathscr{F}^{-1}\left[e^{-t a \cdot \xi} \hat{f}\right] \quad \text { for any } t \in \mathbb{R}, a \in \mathbb{R}^{n}
$$

and $\mathscr{F}^{-1}$ is the Fourier inverse transform:

$$
\mathscr{F}^{-1}[f](\xi)=\frac{1}{(2 \pi)^{n / 2}} \int_{\mathbb{R}^{n}} e^{i x \cdot \xi} f(x) d x .
$$

For $m>0$ and an open interval $I \subset \mathbb{R}$, we define an analytic space:

$$
A_{m}(I) \equiv\left\{f \in L^{\theta}\left(I ; L^{q}\right) ;\|f\|_{A_{m}(I)} \equiv \sup _{a \in B_{1}(0)}\left\|e^{m a \cdot J_{m}} f\right\|_{L^{\theta}\left(I ; L^{q}\right)}<\infty\right\} .
$$

The function space $A_{m}(I)$ with weight $e^{a \cdot x}$ has already been introduced in papers by Hayashi-Ozawa [6] (in space dimension $n=1$ with open interval like 
as $D=(-a, a)$ ) and [12] (in space dimension $n \geq 1$ with symmetric domain $D \subset \mathbb{R}^{n}$ such that $\left.0 \in D,-D=D\right)$.

We denote $e^{m a \cdot J_{m}(t)}$ for $m=\left(m_{1}, \ldots, m_{k}\right) \in \mathbb{Z}_{\geq 0}^{k}$ as follows:

$$
e^{m a \cdot J_{m}(t)} f \equiv\left(e^{m_{1} a \cdot J_{m_{1}}(t)} f_{1}, \ldots, e^{m_{k} a \cdot J_{m_{k}}(t)} f_{k}\right)
$$

for any $f: \mathbb{R}^{n} \rightarrow \mathbb{C}^{k}$.

Our main results are the following.

Theorem 1. Let $1 \leq n \leq 4,1<p \leq 1+\frac{4}{n}$ and $m_{j}>0$. Assume that the nonlinearities $\left\{f_{j}(u, \bar{u})\right\}_{1 \leq j \leq k}$ given by (1.7) satisfy the condition (1.8) and (1.9). Then for any $\phi \in \prod_{j=1}^{k} L_{\exp , m_{j}}^{2}\left(\mathbb{R}^{n}\right)$, there exists $T>0$ and a unique solution $u \in$ $\prod_{j=1}^{k} A_{m_{j}}((-T, T))$ to $(1.1)$. In particular, the solution $u_{j}(t, x)$ is real analytic in $x \in \mathbb{R}^{n}$ for all $t \in(-T, 0) \cup(0, T)$ and any $j=1,2, \ldots, k$.

Since we consider the $L^{2}$ solution of the Cauchy problem (1.1), we need to restrict $1<p \leq 1+\frac{4}{n}$ (cf. Tsutsumi [20]). In order to obtain the analytic smoothing effect for the solution to (1.1), the power of the nonlinearity is restricted to a natural number. Hence the spatial dimension $n$ is naturally restricted to $n=1,2,3,4$. Hoshino [8] showed the existence for the analytic solution to (1.1) with second order nonlinear monomial for 2-component case. Theorem 1 implies the existence for the analytic solution to (1.1) with nonlinear $p$-th order monomial in lower space dimensions and an extension for $k$-system. If we relax the regularity of initial data, then the result can be extended into higher dimensional case.

TheOREM 2. Let $\alpha \in \mathbb{N}, 1 \leq n \leq 2 \alpha+4 /(p-1)$, and $p \in \mathbb{N}$ such that $p<\infty$ if $n \leq 2 \alpha, p \leq 1+4 /(n-2 \alpha)$ if $n>2 \alpha$. Then for any $\phi \in \prod_{j=1}^{k} H_{\exp , m_{j}}^{\alpha}\left(\mathbb{R}^{n}\right)$, there exists $T>0$ and a unique solution $u \in \prod_{j=1}^{k} A_{m_{j}}((-T, T))$ to (1.1) with the nonlinearities given by (1.7) satisfying (1.8) and (1.9). In particular, the solution $u_{j}(t, x)$ is real analytic in $x \in \mathbb{R}^{n}$ for all $t \in(-T, 0) \cup(0, T)$ and any $j=1,2, \ldots, k$.

Remark. All the results by Hoshino-Ozawa [13] involving (1.3), (1.4) and (1.5) can be covered by Theorem 1 under the restriction $p=3$ for the gauge invariant monomials $\left\{f_{1}, f_{2}\right\}$ satisfying (1.6). In [13], they proved the existence of a global analytic solution to the Cauchy problem (1.2) for small data in higher dimension. On the other hand we proved the existence of an analytic solution to (1.2) for a large initial data in lower dimensional cases and our Theorem 1 also extends the known result by Hoshino [8].

In order to show the analytic smoothing effect for the solutions of (1.2), we introduce the operator $e^{m a \cdot J_{m}}$. For each fixed $a \in B_{1}(0)$, the integrability of $e^{m a \cdot J_{m}} f$ implies real analyticity to $a$-direction of a function $f \in D\left(e^{m a \cdot J_{m}}\right)$. Therefore, any function $f \in A_{m}((-T, T))$ is real analytic in $x \in B_{t a}(0)$ for 
$t \in(-T, 0) \cup(0, T)$. We employ the norm; $\sup _{a \in B_{1}(0)}\left\|e^{m a \cdot J_{m}} u\right\|_{L^{\theta}\left(0, T ; L^{q}\right)}$ and it plays an substituted role of the norm $\sum_{k=1}^{\infty} \frac{1}{k !}\left\|\left(J_{m}\right)^{k} u\right\|_{L^{\theta}\left(0, T ; L^{q}\right)}$, where the norm $\|\cdot\|_{L^{\theta}\left(0, T ; L^{q}\right)}$ is the Bochner norm with the $L^{2}$-Strichartz admissible $n / q+2 / \theta=n / 2$ fullfilled. Besides the operator $e^{m a \cdot J_{m}}$ commutes with the free Schrödinger operator $\mathscr{L}_{m}=i \partial_{t}+\frac{1}{2 m} \Delta$ and satisfies the Leibniz rule for the gauge invariant nonlinearities in (1.1). These properties of the operator $e^{m a \cdot J_{m}}$ simplify the proof of Theorem 1 . Because of a commutative relation $\left[\mathscr{L}_{m}, J_{m}\right]=0$, operating $J_{m}^{\alpha}$ with multi-index $\alpha \in \mathbb{Z}_{\geq 0}^{n}$ to the linear Schrödinger equation:

$$
i \partial_{t} v+\frac{1}{2 m} \Delta v=0
$$

and taking summation in $\alpha$, we have the analyticity for the solution to (1.12). We need to show an estimate for the nonlinear term in order to obtain the analyticity for the solution to the Cauchy problem (1.1). We estimate the gauge invariant nonlinearity in $(1.1)$ by using the Galilei transform $J_{m}(t)=$ $x+\frac{i t}{m} \nabla$ that satisfies chain rule for the nonlinear term $|u|^{p-1} u$ :

$$
J_{m}(t)\left(|u|^{p-1} u\right)=\frac{p+1}{2}|u|^{p-1}\left(J_{m}(t) u\right)-\frac{p-1}{2}|u|^{p-3} u^{2}\left(\overline{J_{m}(t) u}\right)
$$

where $p \in \mathbb{N}$ and $m>0$. In the case of $k$-component, the condition (1.6) enables us to compute for the nonlinear term $\left\{f_{j}\right\}_{1 \leq j \leq k}$. Then we introduce a new unknown function $u_{j, a} \equiv e^{m_{j} a \cdot J_{m_{j}}} u_{j}$ (see Section 3 below) and it naturally satisfies the similar system:

$$
\left\{\begin{array}{l}
i \partial_{t} u_{j, a}+\frac{1}{2 m_{j}} \Delta u_{j, a}=f_{j}\left(u_{j, a}, u_{j,-a}\right), \quad j=1,2, \ldots, k, k \in \mathbb{N}, \\
u_{j, a}(0)=e^{m_{j} a \cdot x} \phi_{j},
\end{array}\right.
$$

where $f_{j}\left(u_{j, a}, u_{j,-a}\right)$ is a monomial for a unknown function $u_{j, a}$ and $u_{j,-a}$. Solving the system (1.13) in a proper function space, we obtain that the corresponding solution $u$ also maintains the desired regularity on $a$ direction. Then choosing $a \in B_{1}(0)$ for all direction, we can show that the original solution to (1.1) has real analytic regularity in space variable. Therefore, we have the analytic smoothing effect for the solution to the Cauchy problem (1.1).

\section{Preliminary}

In this section, we state some properties of operator $e^{m a \cdot J_{m}}$ and some basic inequalities. For any set of functions $u=\left(u_{1}, \ldots, u_{k}\right)$ and $v=\left(v_{1}, \ldots, v_{k}\right)$ : 
$\mathbb{R}^{n} \rightarrow \mathbb{C}^{k}$, we define a function $f: \mathbb{C}^{2 k} \rightarrow \mathbb{C}$ as follows:

$$
f(u, v) \equiv \lambda \prod_{j=1}^{k} u_{j}^{\alpha_{j}} v_{j}^{\beta_{j}},
$$

where $\lambda \in \mathbb{C}$.

Hoshino-Hyakuna ([9], Lemma 4) proved the following Lemmas 1 and 2 .

Lemma 1 ([9]). Let $\left(\alpha_{1}, \ldots, \alpha_{k}\right),\left(\beta_{1}, \ldots, \beta_{k}\right) \in \mathbb{Z}_{\geq 0}^{k}$, and $f$ be the $p$-th order monomial given by (2.14). Then for any $y \in \mathbb{R}^{n}$, it holds that

$$
e^{i y \cdot \nabla} f(u, \bar{u})=f\left(e^{i y \cdot \nabla} u, \overline{e^{-i y \cdot \nabla} u}\right) \quad \text { for any } u \in D\left(e^{i y \cdot \nabla}\right),
$$

where $\bar{u}$ denotes as complex conjugate of $u$.

Lemma 2 ([9]). Let $p \in \mathbb{N}, m>0, a \in B_{1}(0),\left(\alpha_{j, \ell}\right)_{1 \leq \ell \leq k},\left(\beta_{j, \ell}\right)_{1 \leq \ell \leq k} \in$ $\mathbb{Z}_{\geq 0}^{k}$ and $f=\left(f_{1}, \ldots, f_{k}\right)$ be a pair of the $p$-th order monomial with the condition (1.9) given by (1.7). Then for any mass coefficient $m=\left(m_{1}, \ldots, m_{k}\right) \in \mathbb{N}^{k}$ satisfying the condition (1.9), it holds that for $j=1,2, \ldots, k$,

$$
e^{m_{j} a \cdot J_{m_{j}}(t)} f_{j}(u, \bar{u})=f_{j}\left(e^{m a \cdot J_{m}(t)} u, \overline{e^{-m a \cdot J_{m}(t)} u}\right)
$$

for any $t \in \mathbb{R}$ and $u \in D\left(e^{m a \cdot J_{m}}\right)$, where $e^{m a \cdot J_{m}(t)} u$ and $e^{-m a \cdot J_{m}(t)} u$ are defined by (1.11).

Stein-Weiss [19] proved the following Lemma 3. The essence of Lemma 3 is similar to that of Lemma A.1 in [12].

LEMMA 3 ([12], [19]). Let $M>0$ and $f$ satisfy

$$
\left\|e^{i y \cdot \nabla} f\right\|_{L^{2}} \leq M, \quad \text { for any } y \in B_{1}(0) .
$$

Then

$$
e^{i y \cdot \nabla} f=(2 \pi)^{-n / 2} \int_{\mathbb{R}^{n}} e^{i(x+i y) \cdot \xi} \hat{f}(\xi) d \xi
$$

is analytic in $\mathbb{R}^{n}+i B_{1}(0)=\left\{x+i y ; x \in \mathbb{R}^{n}, y \in B_{1}(0)\right\}$.

Lemma 4. Let $n \geq 1, m>0$, and $(\theta, q)$ be an admissible pair. Then there exists $C_{0}=C_{0}(n, q)$ such that for any $f \in L^{2}\left(\mathbb{R}^{n}\right)$,

$$
\left\|e^{(i / 2 m) t \Delta} f\right\|_{L^{\theta}\left(-T, T ; L^{q}\right)} \leq C_{0}\|f\|_{L^{2}} .
$$


Moreover, for any $T>0$,

$$
\left\|\int_{0}^{T} e^{(i / 2 m)(t-s) \Delta} f(s) d s\right\|_{L^{2}} \leq C_{0}\|f\|_{L^{\theta^{\prime}\left(-T, T ; L^{q^{\prime}}\right)}},
$$

where $p^{\prime}$ is the dual exponent to $p$ defined by $1 / p+1 / p^{\prime}=1$.

See for the proof, Ginibre-Velo [3], Yajima [21], Cazenave-Weissler [1] and Keel-Tao [17].

\section{Proof of Theorem 1}

ProOF. Let $\quad u \equiv\left(u_{1}, \ldots, u_{k}\right), \quad \bar{u} \equiv\left(\overline{u_{1}}, \ldots, \overline{u_{k}}\right): \mathbb{R} \times \mathbb{R}^{n} \rightarrow \mathbb{C}^{k}, \quad u_{j, a} \equiv$ $e^{m_{j} a \cdot J_{m_{j}}} u_{j}$, and an initial data $\phi=\left(\phi_{1}, \ldots, \phi_{k}\right) \in \prod_{j=1}^{k} L_{\exp , m_{j}}^{2}$. We define a $p$-th order function $f(u, v): \mathbb{C}^{k} \times \mathbb{C}^{k} \rightarrow \mathbb{C}^{k}$ by $f(u, v)=\left(f_{1}(u, v), \ldots\right.$, $\left.f_{k}(u, v)\right)$ and

$$
f_{j}(u, v) \equiv \lambda_{j} \prod_{\ell=1}^{k} u_{\ell}^{\alpha_{j, \ell}} v_{\ell}^{\beta_{j, \ell}} \quad \text { for } j=1, \ldots, k,
$$

where $\lambda_{j} \in \mathbb{C}, \alpha_{j}=\left(\alpha_{j, \ell}\right)_{1 \leq \ell \leq k}, \beta_{j}=\left(\beta_{j, \ell}\right)_{1 \leq \ell \leq k}$ and $m=\left(m_{j}\right)_{1 \leq j \leq k} \in \mathbb{Z}_{\geq 0}^{k}$ satisfy the condition (1.8) and (1.9). We operate $e^{m_{j} a \cdot J_{m_{j}}}$ to the $j$-th equation in (1.1). Then, by using the commutative relation $\left[J_{m_{j}}(t), i \partial_{t}+\frac{1}{2 m_{j}} \Delta\right]=0$ and the identity (2.15) in Lemma 2, we see that

$$
\left\{\begin{array}{l}
i \partial_{t} u_{j, a}+\frac{1}{2 m_{j}} \Delta u_{j, a}=f_{j}\left(u_{j, a}, u_{j,-a}\right), \quad j=1,2, \ldots, k, k \in \mathbb{N}, \\
u_{j, a}(0)=e^{m_{j} a \cdot x} \phi_{j} .
\end{array}\right.
$$

In order to show the analytic smoothing effect for the solution, we solve the following system of integral equations on $L^{\theta}\left(-T, T ; L^{q}\right)$ :

$$
u_{j}=e^{\left(i / 2 m_{j}\right) t \Delta} \phi_{j}-i \lambda_{j} \int_{0}^{t} e^{\left(i / 2 m_{j}\right)(t-s) \Delta} \prod_{\ell=1}^{k} u_{\ell}^{\alpha_{j, \ell}} \bar{u}_{\ell}^{\beta_{j, \ell}} d s
$$

for $j=1, \ldots, k$. Let $I \equiv(-T, T)$ and $M \equiv 4 k C_{0}\|\phi\|_{L_{\mathrm{exp}, m}^{2}}$, where $C_{0}>0$ depends only on the dimension $n$ and exponent $q \geq 2$ in the Strichartz estimate (2.16) and (2.17). We define the metric space

$$
\begin{gathered}
X_{M}(I) \equiv\left\{u=\left(u_{1}, \ldots, u_{k}\right) \in \prod_{j=1}^{k} A_{m_{j}}(I) ; \sum_{j=1}^{k}\left(\left\|u_{j}\right\|_{L^{\theta}\left(I ; L^{q}\right)}+\left\|u_{j}\right\|_{A_{m_{j}}(I)}\right) \leq M\right. \\
\quad \text { for any admissible pair }(\theta, q)\},
\end{gathered}
$$


with the metric

$$
d(u, v) \equiv \sum_{j=1}^{k}\left(\left\|u_{j}-v_{j}\right\|_{L^{\theta}\left(I ; L^{q}\right)}+\left\|u_{j}-v_{j}\right\|_{A_{m_{j}}(I)}\right)
$$

for some admissible pair $(\theta, q)$. We see that $\left(X_{M}(I), d\right)$ is a complete metric space. Therefore we define the operator $\Phi[u]=\left(\Phi_{1}\left[u_{1}\right], \ldots, \Phi_{k}\left[u_{k}\right]\right)$ on $X_{M}(I)$ by

$$
\Phi_{j}\left[u_{j}\right] \equiv e^{\left(i / 2 m_{j}\right) t \Delta} \phi_{j}-i \int_{0}^{t} e^{\left(i / 2 m_{j}\right)(t-s) 4} f_{j}(u, \bar{u}) d s
$$

for $j=1, \ldots, k$ and apply Banach's fixed point theorem to the operator $\Phi$ defined by (3.19). For $1 \leq n \leq 4$, by the Strichartz estimates (2.16), (2.17) and the representation for the operator defined by (1.10), we can estimate for $\Phi_{j}$

$$
\begin{aligned}
\left\|e^{m_{j} a \cdot J_{m_{j}}} \Phi_{j}\left[u_{j}\right]\right\|_{L^{\theta}\left(I ; L^{q}\right)} \leq & \left\|\left(e^{\left(i / 2 m_{j}\right) t \Delta} e^{m_{j} a \cdot x} e^{-\left(i / 2 m_{j}\right) t \Delta}\right) e^{\left(i / 2 m_{j}\right) t \Delta} \phi_{j}\right\|_{L^{\theta}\left(I ; L^{q}\right)} \\
& +\left\|\int_{0}^{t} e^{\left(i / 2 m_{j}\right)(t-s) \Delta} e^{m_{j} a \cdot J_{m_{j}}} f_{j}(u, \bar{u}) d s\right\|_{L^{\theta}\left(I ; L^{q}\right)} \\
\equiv & L_{j, 1}+L_{j, 2}
\end{aligned}
$$

where $L_{j, 1}$ and $L_{j, 2}$ are the linear part and the Duhamel part of the integral equation (3.19), respectively. By the Strichartz estimate (2.16), we have

$$
L_{j, 1}=\left\|e^{\left(i / 2 m_{j}\right) t \Delta} e^{m_{j} a \cdot x} \phi_{j}\right\|_{L^{\theta}\left(I ; L^{q}\right)} \leq C_{0}\left\|e^{m_{j} a \cdot x} \phi_{j}\right\|_{L^{2}} \leq \frac{1}{4 k} M .
$$

Similarly applying (2.17) to $L_{j, 2}$ with admissible pair $(4 q /(4 q+n q-2 n p), q / p)$ and using Hölder's inequality, we have

$$
\begin{aligned}
L_{j, 2} & \leq C_{0}\left\|\prod_{\ell=1}^{k}\left(e^{m_{\ell} a \cdot J_{m_{\ell}}} u_{\ell}\right)^{\alpha_{j, \ell}}\left(\overline{e^{-m_{\ell} a \cdot J_{m_{\ell}}} u_{\ell}}\right)^{\beta_{j, \ell}}\right\| \|_{L^{4 q /(4 q+n q-2 n p)\left(I ; L^{q / p}\right)}} \\
& \leq C_{0}\left\|\prod_{\ell=1}^{k}\right\| e^{m_{\ell} a \cdot J_{m_{\ell}}} u_{\ell}\left\|_{L^{q}}^{\alpha_{j, \ell}}\right\| e^{-m_{\ell} a \cdot J_{m_{\ell}}} u_{\ell}\left\|_{L^{q}}^{\beta_{j, \ell}}\right\|_{L^{4 q /(4 q+n q-2 n p)(I)}} \\
& \leq C_{0} T^{1-(n / 4)(p-1)} \prod_{\ell=1}^{k}\left\|e^{m_{\ell} a \cdot J_{m_{\ell}}} u_{\ell}\right\|_{L^{\theta}\left(I ; L^{q}\right)}^{\alpha_{j, \ell}}\left\|e^{-m_{\ell} \cdot J_{m_{\ell}}} u_{\ell}\right\|_{L^{\theta}\left(I ; L^{q}\right)}^{\beta_{j, \ell}} \\
& \leq C_{0} T^{1-(n / 4)(p-1)} M^{p} .
\end{aligned}
$$


Conbining (3.20), (3.21) and (3.22), we have

$$
\left\|\Phi_{j}\left[u_{j}\right]\right\|_{A_{m_{j}}(I)} \leq \frac{1}{4 k} M+C_{0} T^{1-(n / 4)(p-1)} M^{p} .
$$

In the same way as above, we have that for $u, v \in X_{M}(I)$,

$$
\begin{aligned}
& \left\|e^{m_{j} a \cdot J_{m_{j}}}\left(\Phi_{j}\left[u_{j}\right]-\Phi_{j}\left[v_{j}\right]\right)\right\|_{L^{\theta}\left(I ; L^{q}\right)} \\
& \quad \leq\left\|\int_{0}^{t} e^{\left(i / 2 m_{j}\right)(t-s) \Delta} e^{m_{j} a \cdot J_{m_{j}}}\left(f_{j}(u, \bar{u})-f_{j}(v, \bar{v})\right) d s\right\|_{L^{\theta}\left(I ; L^{q}\right)} \\
& \quad \leq C_{0} T^{1-(n / 4)(p-1)} M^{p-1} \sum_{j=1}^{k}\left\|u_{j}-v_{j}\right\|_{A_{m_{j}}(I)} .
\end{aligned}
$$

Hence we obtain

$$
d(\Phi[u], \Phi[v]) \leq C_{0} T^{1-(n / 4)(p-1)} M^{p-1} d(u, v) .
$$

Choosing $T>0$ such that

$$
\left\{\begin{array}{l}
C_{0} T^{1-(n / 4)(p-1)} M^{p} \leq \frac{1}{4 k} M, \\
C_{0} T^{1-(n / 4)(p-1)} M^{p-1} \leq \frac{1}{2},
\end{array}\right.
$$

we have that the map $\Phi$ is a contraction over $X_{M}(I)$. Then Banach's fixed point theorem implies that $\Phi$ has a unique fixed point $u$ in $X_{M}(I)$ which is a solution of the integral equation (3.19). In other words, the solution $u=$ $\left(u_{1}, \ldots, u_{k}\right)$ satisfies

$$
\sup _{a \in B_{1}(0)}\left\|e^{m_{j} a \cdot J_{m_{j}}} u_{j}\right\|_{L^{\theta}\left(I ; L^{q}\right)}=\sup _{a \in B_{1}(0)}\left\|e^{i t a \cdot \nabla} e^{-\left(i m_{j}|x|^{2} / 2 t\right)} u_{j}\right\|_{L^{\theta}\left(I ; L^{q}\right)}<\infty
$$

for any $a \in B_{1}(0)$ and $j=1, \ldots, k$. Therefore Lemma 3 yields that $e^{-i m_{j}|x|^{2} / 2 t} u_{j}$ is analytic over $\mathbb{R}^{n}+i B_{t}(0)$. Since $e^{-i m_{j}|x|^{2} / 2 t}$ is analytic over $\mathbb{R}^{n}+i \mathbb{R}^{n}, u$ is real analytic in $x \in \mathbb{R}^{n}$.

In the critical case for $n=4$, we define

$$
M=\sup _{a \in B_{1}(0)}\left\|e^{\left(i t / 2 m_{j}\right) \Delta} e^{m_{j} a \cdot x} \phi\right\|_{L^{\theta}\left(I ; L^{q}\right)}
$$

in the space $X_{M}(I)$ defined by (3.18). Taking sufficiently small $T>0$, we can construct the local solution $v=e^{m a \cdot J_{m}} u$ in the Stricartz space $L^{\theta}\left(I ; L^{q}\right)$ by applying Banach's fixed point theorem. 
The same argument for the proof of Theorem 1 can be applied to the proof of Theorem 2 .

\section{Acknowledgments}

The authors express their thanks to the referees and the editor for their helpful suggestions. The first author is supported by Grant-in-aid for Scientific Research A, No. 19H00638.

\section{References}

[1] Cazenave, T., Weissler, F., The Cauchy problem for the nonlinear Schrödinger equation in $H^{1}$, Manuscripta Math. 61 (1988), no. 4, 477-494.

[2] De Bouard, A., Analytic solutions to nonelliptic nonlinear Schrödinger equations, J. Differential Equations 104 (1993), no. 1, 196-213.

[3] Ginibre, J., Velo, G., On a class of nonlinear Schrödinger equations. I. The Cauchy problem, general case, J. Funct. Anal. 32 (1979), no. 1, 1-31.

[4] Hayashi, N., Saitoh, S., Analyticity and smoothing effect for the Schrödinger equation, Ann. Inst. Henri Poincaré, Phys. Théor. 52 (1990), 163-173.

[5] Hayashi, N., Saitoh, S., Analyticity and global existence of small solutions to some nonlinear Schrödinger equation, Commun. Math. Phys. 129 (1990), $27-41$.

[6] Hayashi, N., Ozawa, T., On the derivative nonlinear Schrödinger equation, Phys. D. 55 (1992), no. 1-2, 14-36.

[7] Hoshino, G., Analyticity of solutions to a quadratic system of Schrödinger equations without mass resonance condition, J. Math. Anal. Appl. 467 (2018), no. 1, 331-348.

[8] Hoshino, G., Analytic smoothing effect for global solutions to a quadratic system of nonlinear Schrödinger equations, Nonlinear Differential Equations Appl. 24 (2017), no. 6, Art. 62, 17.

[9] Hoshino, G., Hyakuna, R., Analytic smoothing effect for the nonlinear Schrödinger equations without square integrability, J. Fourier Anal. Appl. 24 (2018), no. 6, 1661-1680.

[10] Hoshino, G., Ozawa, T., Analytic smoothing effect for a system of nonlinear Schrödinger equations, Differ. Equ. Appl. 5 (2013), 395-408.

[11] Hoshino, G., Ozawa, T., Analytic smoothing effect for nonlinear Schrödinger equation in two space dimensions, Osaka J. Math. 51 (2014), 609-618.

[12] Hoshino, G., Ozawa, T., Analytic smoothing effect for nonlinear Schrödinger equations with quintic nonlinearity, J. Math. Anal. Appl. 419 (2014), 285-297.

[13] Hoshino, G., Ozawa, T., Analytic smoothing effect for a system of nonlinear Schrödinger equations with two wave intaraction, Adv. Differential Equations 20 (2015), 697-716.

[14] Kato, T., On nonlinear Schrödinger equations, Ann. Inst. H. Poincaré Phys. Théor 46 (1987), 113-129.

[15] Kato, K., Ogawa, T., Analyticity and smoothing effect for the Korteweg-de Vries equation, Sūrikaisekikenkyūsho Kōkyūroku 1407 (1998), 47-55.

[16] Kato, K., Ogawa, T., Analyticity and smoothing effect for the Korteweg de Vries equation with a single point singularity, Math. Ann. 316 (2000), 577-608.

[17] Keel, M., Tao, T., Endpoint Strichartz estimates, Amer. J. Math. 120 (1998), no. 5, 955-980. 
[18] Nakamitsu, K., Analytic finite energy solutions of the nonlinear Schrödinger equation, Commun. Math. Phys. 260 (2005), 117-130.

[19] Stein, E., Weiss, M., Introduction to Fourier analysis on Euclidian spaces, Princeton Landmarks in Mathematics, Princeton University Press, Princeton NJ, (1971).

[20] Tsutsumi, Y., $L^{2}$ solution for nonlinear Schrödinger equation and nonlinear groups, Funk. Ekvac. 30 (1987), 115-125.

[21] Yajima, K., Existence of solutions for Schrödinger evolution equations, Commun. Math. Phys. 110 (1987), 415-426.

\author{
Takayoshi Ogawa \\ Mathematical Institute/ \\ Research Alliance Center of Mathematical Science \\ Tohoku University \\ Sendai 980-8578, Japan \\ E-mail: ogawa@math.tohoku.ac.jp
}

Takuya Sato

Mathematical Institute, Tohoku University

Sendai 980-8578, Japan

E-mail: takuya.sato.t7@dc.tohoku.ac.jp 